\title{
Scientific Performance of a Nano-satellite MeV Telescope
}

\author{
Giulio Lucchetta ${ }^{1,3}$, Francesco Berlato ${ }^{1,4}$, Riccardo Rando ${ }^{1,2}$, Denis Bastieri ${ }^{1,2}$, and Giorgio Urso ${ }^{1}$ \\ ${ }^{1}$ Dipartimento di Fisica \& Astronomia “G. Galilei,” Università di Padova, I-35131 Padova, Italy; giulio.lucchetta@desy.de, fberlato@mpe.mpg.de \\ ${ }^{2}$ Istituto Nazionale di Fisica Nucleare, Sezione di Padova, I-35131 Padova, Italy \\ Received 2017 February 6; revised 2017 March 10; accepted 2017 March 27; published 2017 April 28
}

\begin{abstract}
Over the past two decades, both X-ray and gamma-ray astronomy have experienced great progress. However, the region of the electromagnetic spectrum around $\sim 1 \mathrm{MeV}$ is not so thoroughly explored. Future medium-sized gamma-ray telescopes will fill this gap in observations. As the timescale for the development and launch of a medium-class mission is $\sim 10$ years, with substantial costs, we propose a different approach for the immediate future. In this paper, we evaluate the viability of a much smaller and cheaper detector: a nano-satellite Compton telescope, based on the CubeSat architecture. The scientific performance of this telescope would be well below that of the instrument expected for the future larger missions; however, via simulations, we estimate that such a compact telescope will achieve a performance similar to that of COMPTEL.
\end{abstract}

Key words: astroparticle physics - gamma rays: general - instrumentation: detectors

\section{Introduction}

High-energy astrophysics focuses specifically on space telescopes and ground observatories. At very high energies $(>500 \mathrm{GeV})$ ground-based Cherenkov telescopes are the instrument of choice (Horns 2016), and the new observatory CTA is under construction (see Acharya et al. 2013). At lower energies, Fermi-LAT and AGILE observe the energy range from $\sim 40 \mathrm{MeV}$ to $2 \mathrm{TeV}$ (Tavani et al. 2008; Atwood et al. 2009), and since their launch, they have significantly contributed to the research in this energy band. A variety of $\mathrm{X}$-ray and hard X-ray observatories covers the lower energies, for example: NuSTAR (Harrison et al. 2013), Swift (Gehrels et al. 2004), or INTEGRAL (Winkler et al. 2011). In addition, the DAMPE satellite, even if its main scientific target is Cosmic Rays, will also provide useful information about gamma-rays (Chang 2014).

This leaves a relatively poorly explored part of the electromagnetic spectrum around $\sim 1 \mathrm{MeV}$. The COMPTEL telescope, on board CGRO (see Schönfelder et al. 1993), was the last detector to observe this region, with the sensitivity shown in Figure 1. Observations in this region are complex, for various reasons. Compton scattering is the dominating interaction of photons with matter, implying a complex event reconstruction process. Moreover, activation due to cosmic radiation causes significant background internally to the instruments. Compton observatories have been proposed for the immediate future (most notably, e-Astrogam, see De Angelis et al. 2017), with costs around half a billion Euros and a timescale for development and launch of $\sim 10$ years. Furthermore, intermediate sized missions have been proposed in recent years, like MEGA (Bloser et al. 2006) and SMILE (Tanimori et al. 2015). The scientific case for a deeper exploration of this energy range is strong, and it is well detailed (see, for example, De Angelis et al. 2017).

On a very short timescale, a nano-sat telescope may be profitable. While the scientific performance would be well below the one expected for larger future missions, it could rival COMPTEL's telescope. The small cost (approximately half a

\footnotetext{
Now at DeSy, Zeuthen.

4 Now at MPE, Garching.
}

million Euros) and rapidity of realization are clear advantages; one satellite could be built and launched in about one year. Such a nano-scale instrument, based on a scientific payload similar to proposed large-scale missions, could also be used as a pathfinder to test technologies and algorithms for employment in later missions. In this work, we evaluate the performance of a nano-satellite $\mathrm{MeV}$ telescope based on the CubeSat standard.

\section{CubeSat Design}

A CubeSat is a standardized model of a miniaturized satellite, with precise restrictions for both volume and weight: it is based on a $10 \times 10 \times 10 \mathrm{~cm}^{3}$ cubic unit with a maximum weight of $1.33 \mathrm{~kg}$ (1U CubeSat). In this paper, we study a payload that is compatible with a $2 \mathrm{U}$ standard. ${ }^{5}$

As shown in Figure 2, the structure of the detector is based on a design that has already been tested by previous missions, such as Fermi-LAT and AGILE: a silicon microstrip tracker is coupled with a $\mathrm{CsI}(\mathrm{Tl})$ calorimeter, enclosed in a plastic anticoincidence shield. Such a configuration performed extremely well for a pair production telescope, and we believe it can be optimized to work effectively in the lower energy range as well (roughly comprised in $0.1 \div 10 \mathrm{MeV}$ ), where Compton interaction prevails. The primary scope of our design is to detect photons through Compton interaction, but the detector is also capable of pair detection, though only to the point where the small size of the calorimeter does not allow for full absorption of high-energy gamma-rays.

Of course, the details of this design will significantly impact the scientific performance. For the first evaluation, we opted for several major simplifications. Notably, in our design we will simulate only active materials, ignoring, from now on, structural elements and the fact that some space will be reserved for the read-out electronics. We assume that we can neglect the effect of the former and that we can simply estimate the effect of the latter at the first order in a later stage, taking into account how many of the simulated events are lost when removing active material.

\footnotetext{
5 We estimate a $4 \mathrm{U}$ model in the final design when the on-board electronics and the flight system are added.
} 


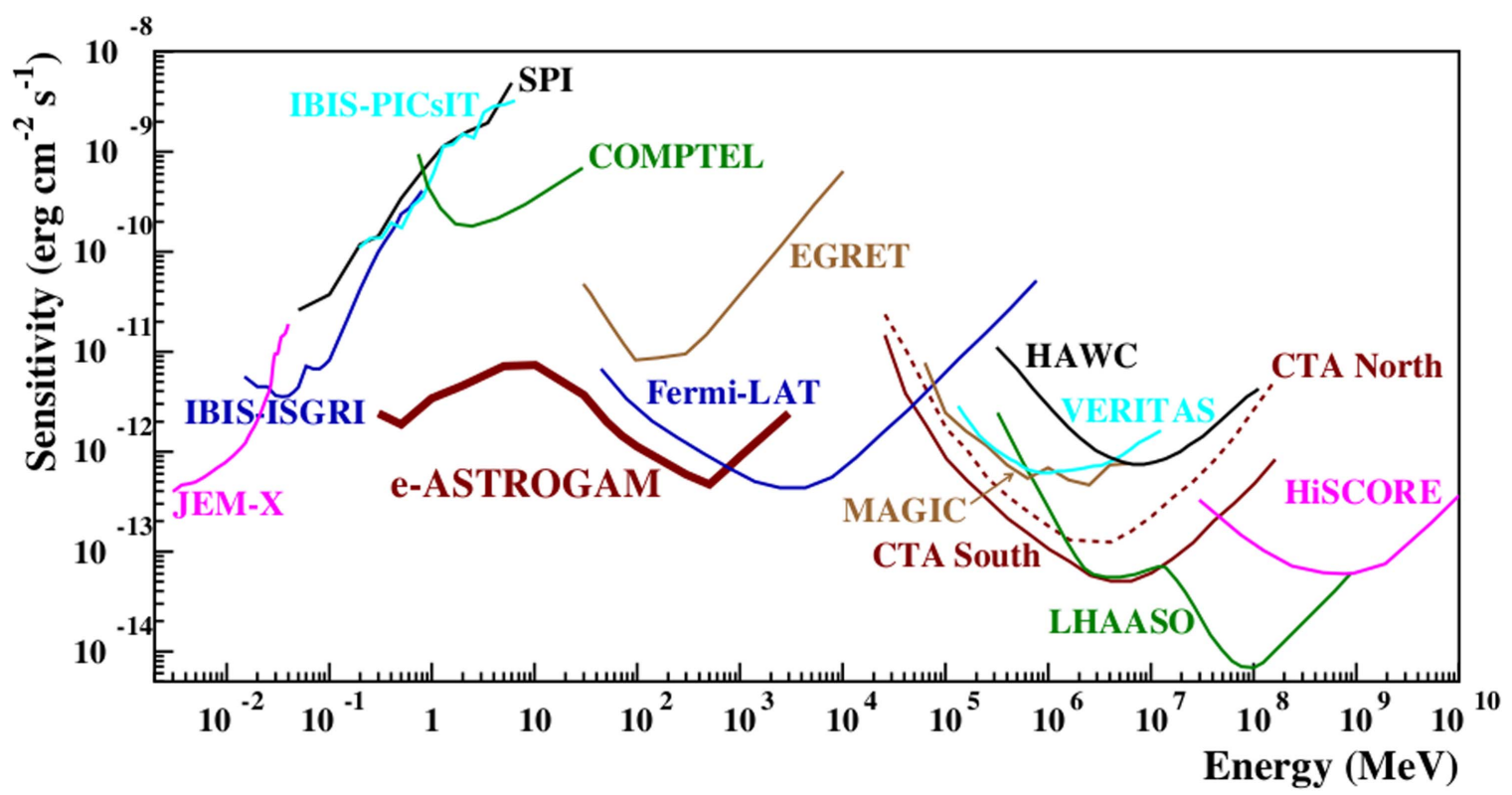

Figure 1. Current sensitivity for the past missions and possible improvement due to e-Astrogam. Image from De Angelis et al. (2017).

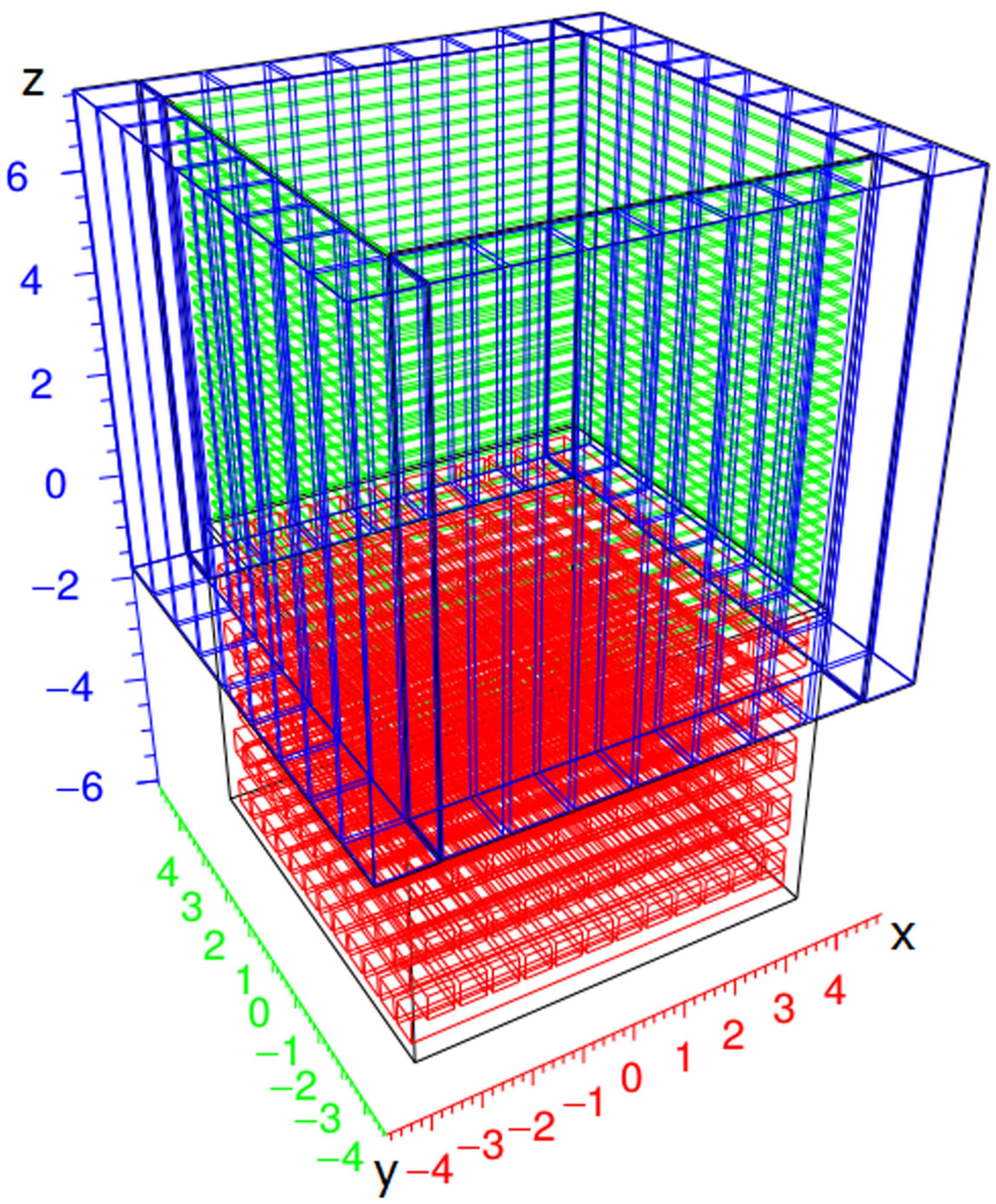

Figure 2. Schematic of the CubeSat geometry (anti-coincidence not shown). 
Table 1

Silicon Tracker Specifications

\begin{tabular}{ll}
\hline \hline Parameter & Value or Range \\
\hline Tracker size & $7.7 \times 7.7 \times 7.5 \mathrm{~cm}^{3}$ \\
Number of layers & 30 \\
Layer thickness & $500 \mu \mathrm{m}$ \\
Strip pitch & $150 \mu \mathrm{m}$ \\
Guard ring & $1 \mathrm{~mm}$ \\
Read-out electronics & VATA460 \\
Electronic noise & $1200 \mathrm{e}^{-}$ \\
Bit digitization & 10 \\
\hline
\end{tabular}

We will now present in more detail the scientific payload and then discuss the simulation performed and results obtained.

\subsection{Tracker}

The ability to track electrons will be the greatest improvement of future Compton telescopes over COMPTEL. In our case, this is possibly due to the use of a silicon microstrip detector. The use of a silicon tracker allows for good spatial resolution, limiting the impact of the short lever arm. Based on the excellent performance of missions like the Fermi-LAT, we know that this choice is suitable for pair detection or higher energy photons in general. However, in the Compton regime, we can reconstruct the track of scattered electrons only in a fraction of the total events (tracked events in our data set: $14 \%$ at $500 \mathrm{keV}, 30 \%$ at $1 \mathrm{MeV}$, and $28 \%$ at $3 \mathrm{MeV}$ ).

We know from AGILE that by using a silicon-strip tracker with an analog read-out, we can achieve a spatial resolution below $40 \mu \mathrm{m}$, compared to the values of $1.5 \div 2.3 \mathrm{~cm}$ achieved by COMPTEL (Schönfelder et al. 1993). Finally, whereas AGILE and Fermi-LAT use Single-Sided Strip Detectors (SSSDs), the tracker for a Compton Telescope needs Double-Sided Strip Detectors (DSSDs) in order to obtain precise information about the electrons' direction.

The tracker's specifications are summarized in Table 1. Regarding the signal read-out, several ASICs can be considered. VATA460 has an acceptable input range of $95 \mathrm{fC}$, adequate shaping time $(\sim 2 \mu \mathrm{s})$, power consumption $(0.3 \mathrm{~mW} / \mathrm{ch})$, and trigger capabilities (Odaka et al. 2012). A 10 bit digitization gives a reading accuracy of $2.1 \mathrm{keV}$, while the equivalent noise charge is around $1200 \mathrm{e}^{-}$, which corresponds to an electronic noise of $\sim 4.3 \mathrm{keV}$. Given these parameters, the energy resolution is $\sim 5 \mathrm{keV}(1 \sigma)$. The impact of the tracker's design on the performance is analyzed in detail in Lucchetta (2016).

\subsection{Calorimeter}

The calorimeter is made of $\mathrm{CsI}(\mathrm{Tl})$ crystals (see Table 2), again, a material that produces good performance, and was already thoroughly tested by previous missions. The crystals are also very cheap compared to the tracker and do not significantly affect the overall cost of the detector. Furthermore, the number of channels for the read-out is small compared to that of the tracker. CsI(Tl) crystals can be easily coupled to a photodiode, given their wavelength of maximum emission and their high photon yield. This allows for a simple, yet effective calorimeter design.

We consider the crystals coupled to a common photodiode read-out (e.g., Hamamatsu S3590). For each keV of absorbed energy, $\sim 360 \mathrm{e}^{-}$would be emitted in the photodiode, and a
Table 2

CsI(Tl) Calorimeters Specifications

\begin{tabular}{ll}
\hline \hline Parameter & Value or Range \\
\hline Bottom crystals size & $0.5 \times 0.5 \times 7.5 \mathrm{~cm}^{3}$ \\
Lateral crystals size & $1.0 \times 1.0 \times 8.5 \mathrm{~cm}^{3}$ \\
Depth resolution $(1 \sigma)$ & $1 \mathrm{~cm}$ \\
Photodiode read-out & Hamamatsu S3590 \\
Read-out electronics & VATA460 \\
Crystals per bottom plane & 12 \\
Bottom planes & 10 \\
\hline
\end{tabular}

reading accuracy of $\sim 2.3 \mathrm{keV}$ would be achieved. Although silicon photomultipliers can achieve a better energy resolution, we chose photodiodes in order to use the same ASIC as in the tracker, a considerable simplification. The impact of the calorimeter's design on the performance is analyzed in detail in Berlato (2016).

In our detector design, there are two calorimeter modules: a bottom one and a lateral one. The latter is particularly important for lower-energy Compton detection, while the former is important for high-energy Compton and pair detection.

In the bottom calorimeter, the layers are arranged in a hodoscopic configuration, with crystals ordered along the $x$ and $y$ axes. Such a setup was chosen to keep any read-out electronics at the edges of the calorimeter. In this way, we will decrease the amount of passive materials the photons encounter along their path as much as possible.

The lateral calorimeter, surrounding the tracker, is made of CsI(Tl) crystals with a section of $1.0 \times 1.0 \mathrm{~cm}^{2}$ and is long enough to have a partial overlap $(\sim 1 \mathrm{~cm})$ with the bottom calorimeter.

We can reasonably assume to achieve a depth resolution for the crystals of at least $1.0 \mathrm{~cm}$.

\subsection{Anti-coincidence Detector (ACD)}

Our design includes an ACD to allow for the rejection of charged background. The ACD is composed of several slabs of plastic scintillator, each $5 \mathrm{~mm}$ thick, read by silicon photomultipliers. Its contained cost, high performance, and reliability have already been proven in various previous missions, see for example Atwood et al. (2009) or Tavani et al. (2008). We can realistically expect a rejection efficiency $\gtrsim 99.99 \%$.

\subsection{Cost of the Scientific Instruments}

We estimate a cost of $\sim 500 \mathrm{k} €$ for the procurement of the sensors and of the read-out ASICs, including spares for tests and contingency. The largest expense is the tracker (90\%), with comparable total costs shared between DSSD and ASICs.

\section{Detector Performance Parameters}

Canonically, the performance of a telescope is characterized in terms of effective area, energy resolution, and angular resolution, all assumed to be independent from one another.

For a Compton telescope, the angular resolution can be somewhat more complex compared to that of the pair production. The angular resolution is defined via two different quantities (as defined in Bloser et al. 2004). The first one, called Angular Resolution Measure (ARM), quantifies how precisely the direction of the scattered photon has been reconstructed. Specifically, it is given by the difference of the real (or 
simulated) and measured scatter angle. The second contribution comes from the Scatter Plane Deviation (SPD). The SPD quantifies how well the electron track is reconstructed and is defined as the difference between the actual scatter plane and the measured one. Adopting the same notation used by Zoglauer (2005), for an untracked event, the SPD is always equal to the maximum value of $180^{\circ}$.

Using both ARM and SPD, we can then define a final angular resolution element on the sky map, which yields the following solid angle $\Delta \Omega$ :

$$
\Delta \Omega=\left[\cos \left(\bar{\varphi}-\sigma_{\mathrm{ARM}}\right)-\cos \left(\bar{\varphi}+\sigma_{\mathrm{ARM}}\right)\right] \cdot 2 \sigma_{\mathrm{SPD}}
$$

where $\bar{\varphi}$ is the average photon scatter angle, $\sigma_{\mathrm{ARM}}$ is the ARM FWHM, and $\sigma_{\text {SPD }}$ is the SPD HWHM.

For pair events, the angular resolution is described in a more straightforward manner and is given by the point-spread function (PSF) as in Ackermann et al. (2013).

As high-level performance for the detector, we consider the continuum sensitivity, which quantifies the telescope's ability to detect a weak source in presence of background. The sensitivity is computed through Poisson statistics, via the following formula (Zoglauer 2005):

$$
F_{S}=\frac{z^{2}+z \sqrt{z^{2}+4 N_{\mathrm{bkg}}}}{2 T_{\mathrm{eff}} A_{\mathrm{eff}}}
$$

where $z$ is the statistical significance imposed in unit of sigmas (that is, the number of Poisson's error bars), $N_{\mathrm{B}}$ is the number of background events, $T_{\text {eff }}$ is the effective observation time, and $A_{\text {eff }}$ is the effective area. $N_{\mathrm{B}}$ can be computed with a semianalytical procedure using the data from simulations, as we will see in the next section.

\section{Simulation Methodology}

In this work, simulations are performed using the software and data analysis tool MegaLib (see Zoglauer et al. 2008).

We simulated an isotropic source with energy comprised in $0.1 \leqslant E \leqslant 10 \mathrm{MeV}$ using a power law with energy index of -1 . This allows equal statistics in each solid angle and logarithmic energy bin.

We then divide data into different event classes. In this investigation, we decided to evaluate the telescope performance simply by dividing the simulated events into tracked and untracked. ${ }^{6}$ Selecting only tracked events allows us to greatly improve the angular resolution element (mainly due to the SPD), but this comes at the expense of the effective area. On the other hand, the use of untracked events grants a greater effective area, but the angular resolution element worsens considerably.

To estimate the sensitivity, one must carefully evaluate the contribution from each source of background events. Because this design, like other Compton telescopes, is meant for a Low Earth Orbit (LEO; $\sim 500 \mathrm{~km}$, equatorial), the main background contributions are (De Angelis et al. 2017) as follows.

1. Extra-galactic background: a diffuse, isotropic, and homogeneous photon background, mainly due to unresolved sources.

\footnotetext{
6 Possible subclasses that could be studied are events with hits either in the lateral or bottom calorimeter.
}

2. Earth's gamma emission: the interaction between primary cosmic rays and the Earth's atmosphere generates secondary particles and, in particular, a bright gammaray flux. Earth's gamma emission represents the most significant background contribution in our design.

3. Charged background: events due to charged cosmic rays hitting the detector. The vast majority of them ( $\gtrsim 99.99 \%)$ can be effectively vetoed through the use of an anticoincidence detector.

4. Activation: the continuous flux of cosmic rays, both charged and uncharged, activates the satellite materials, i.e., produces radioactive isotopes. This source of background depends strongly on the exact materials and geometry and can change the final spectrum considerably. This contribution scales with the amount of passive material in the telescope.

Continuum sensitivity can be estimated through a semianalytical method. Background fluxes are derived from previous missions' data: Earth's gamma emission from De Angelis et al. (2017) and EGB from Georg (1999), extended at lower energies. ${ }^{7}$

Given our energy range and planned orbit, the major contribution to the background due to charged particles is caused by secondary electrons (De Angelis et al. 2017). Our simulations of secondary electrons span several hours, resulting in a few accepted events only_enough to estimate a negligible event rate in our energy range (less than $10^{-4} \mathrm{~Hz}$ with respect to $\sim 1 \mathrm{~Hz}$ for the EGB), but too few to determine the spectrum. This holds when considering the hypothesis of a hermetic ACD, but, in reality, the charged particle background will be entirely determined by the inefficiencies of the ACD assembly, e.g., the seams, which are still unclear at this stage. For this reason, we do not embark now in a major simulation effort and, with the caveats above, we set $N_{\text {chg }}=0$, the number of events due to charged particles.

Then, the total number of background events is given by:

$$
N_{\mathrm{bkg}} \simeq N_{\mathrm{egb}} \cdot(1+R)
$$

where $R$ is the ratio between Earth's gamma emission and EGB events and $N_{\mathrm{egb}}$ is the number of EGB events inside the angular resolution element: $N_{\mathrm{egb}}=F_{\mathrm{egb}} \cdot A_{\mathrm{eff}} \cdot T_{\mathrm{eff}} \cdot \Delta \Omega$. We assumed that the detector is pointing to the zenith (normally outwards from the Earth), and the source is placed at the zenith angle $\Theta=0$, which is the best case scenario for the sensitivity value. Nominally, the flux of the Earth's gamma-rays at angles $\Theta<90^{\circ}$ is null. However, a small fraction of events presents a large SPD, so the Compton arc partially lies in the sky map element we are observing. Because the Earth's gamma emission flux is considerably larger in an LEO, this is still the most significant background source for our design.

This problem was mainly avoided by COMPTEL, which, thanks to fast photomultiplier tubes and the time-of-flight measurements, was able to distinguish upward and downward events.

In our case, this issue may be resolved by implementing some kind of trigger capable of rejecting at least a significant fraction of the Earth's gamma emission events, or by trying to

\footnotetext{
7 As the measured EGB flux depends on resolved sources, and therefore telescope sensitivity, we made use of COMPTEL's data, which performed similarly to ours.
} 


\section{Effective area comparison}

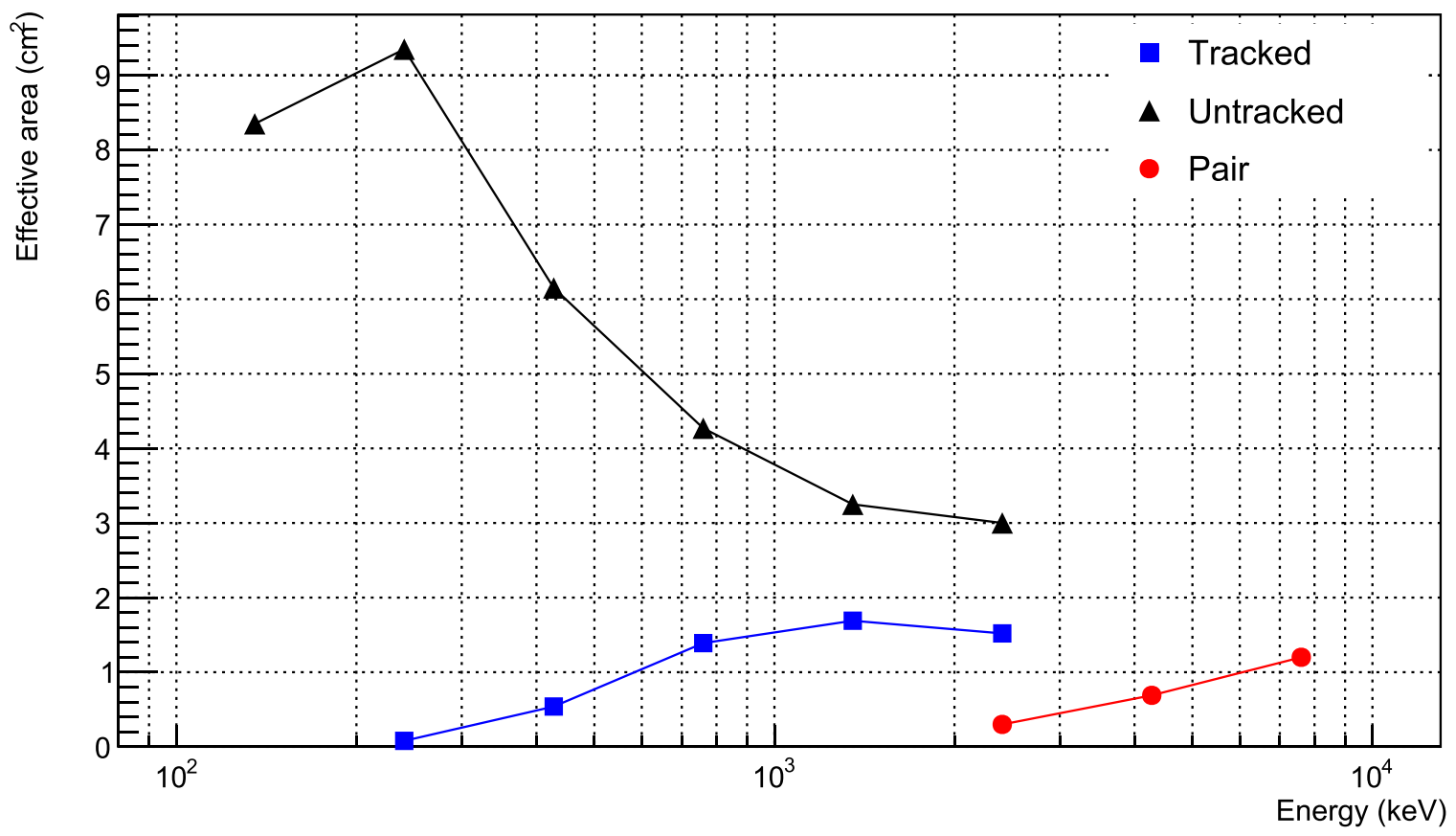

Figure 3. Estimated effective area for Compton (both tracked and untracked) and pair events (at the zenith angle).

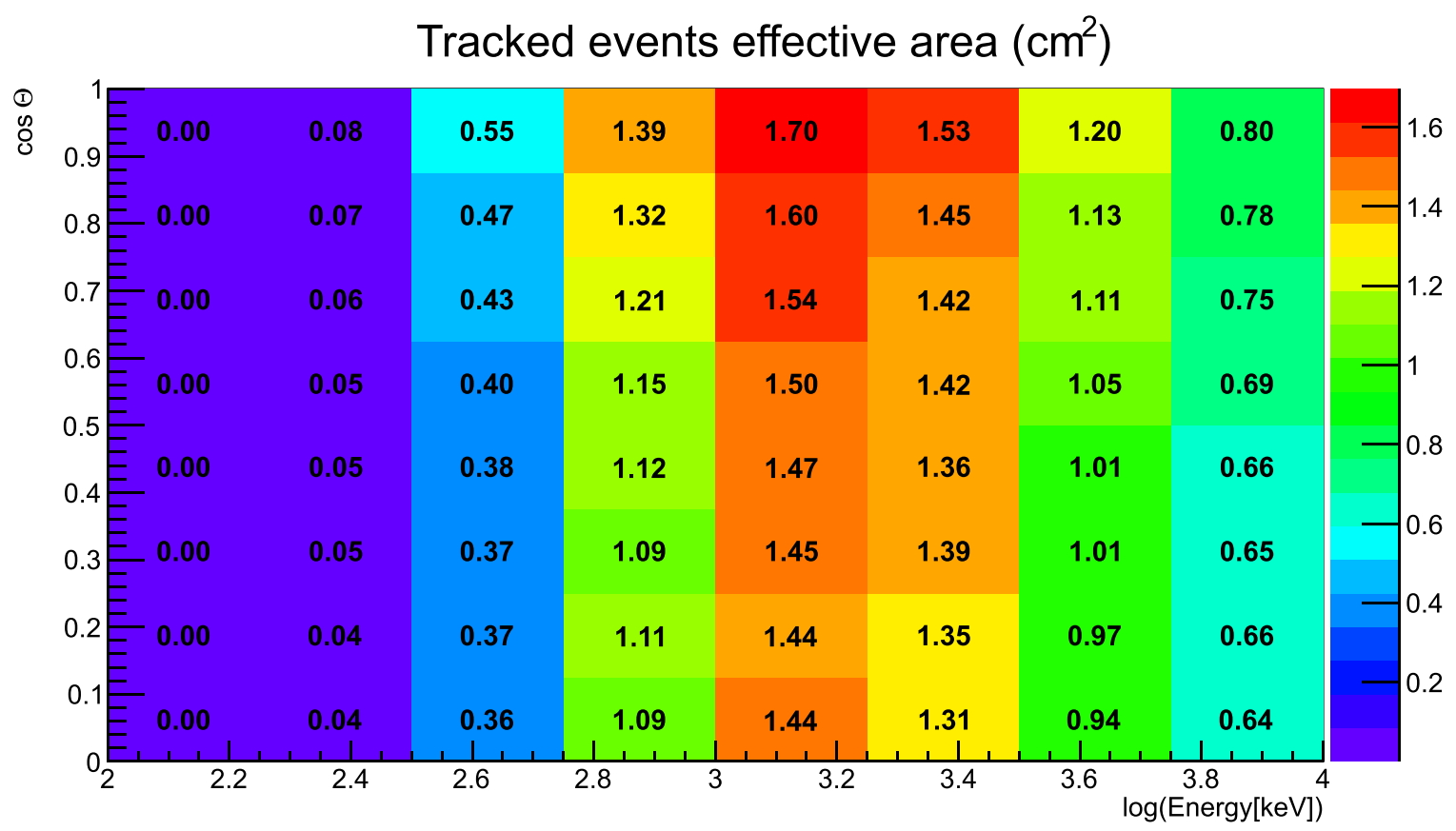

Figure 4. Effective area for tracked events in the function of Monte Carlo energy and direction of the initial photon (cos $\Theta=1$ for normal incidence).

discard them during the data analysis process by means of event cuts.

\section{Performance Estimate}

The first parameter we estimate is the effective area. In Figure 3, we can see the effective area computed at the zenith angle as a function of the incident photon energy, for tracked and untracked Compton events. For the design of the telescope under study, the dependence of the effective area on the polar angle $\Theta$ is rather weak, as can be seen for tracked events in
Figure 4. For example, at simulated energies around $1 \mathrm{MeV}$, the difference of the effective area from normal incidence to an angle of $90^{\circ}$ is less than $15 \%$. This means that the field-of-view is very wide compared to COMPTEL (see Kappadath 1998).

Figure 3 does not show uncertainties: statistical errors are not particularly significant in this phase, as systematic uncertainties are dominant. On the other hand, systematic errors cannot be properly estimated before the final design is established.

The effective area for tracked events is significant only at energies above several hundreds of $\mathrm{keV}$, but the effective area 


\section{Angular resolution plot (comparison)}

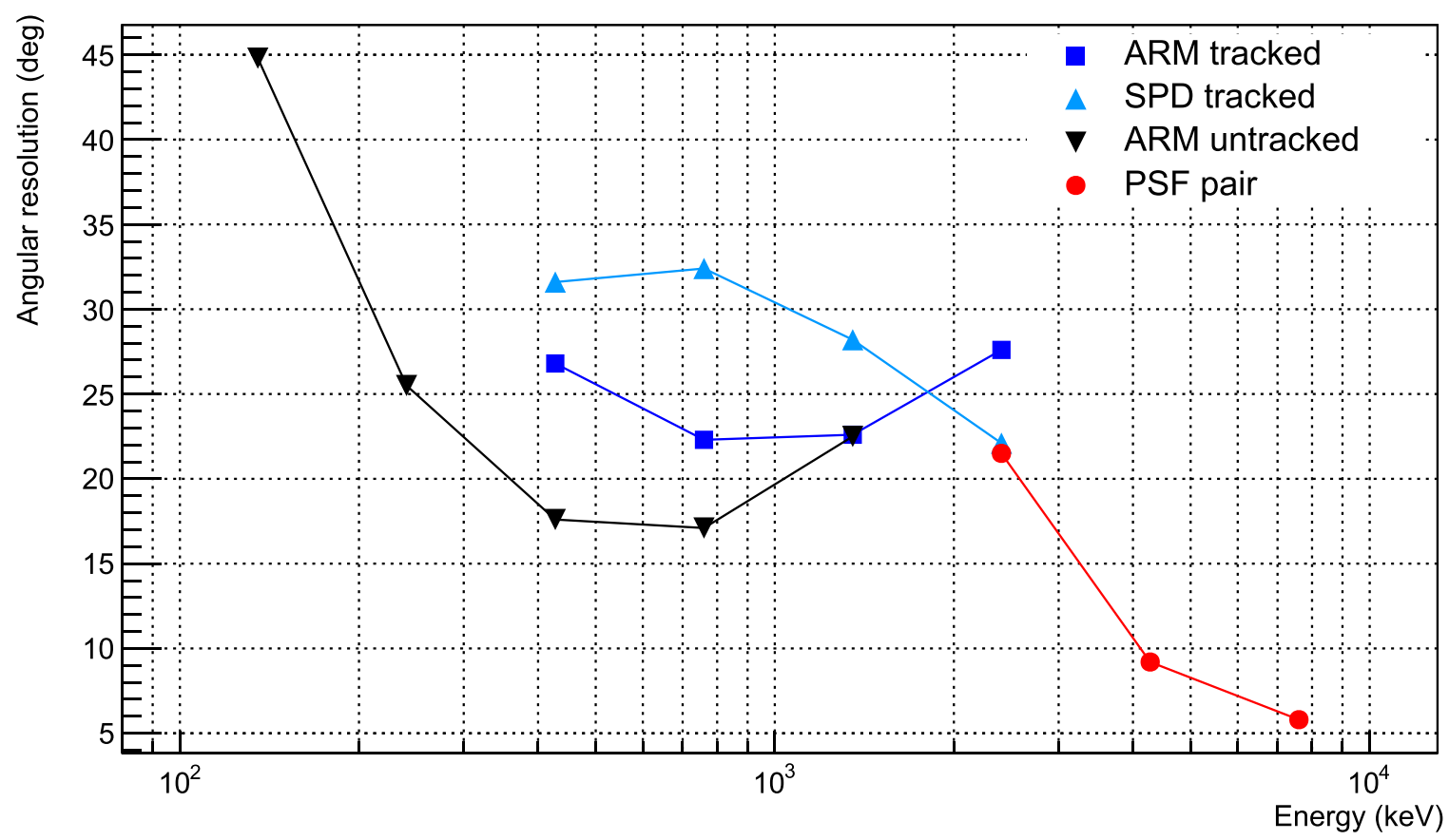

Figure 5. Estimated ARM and SPD for Compton events (both tracked and untracked), and PSF for pair events.

for untracked events peaks around $200 \div 300 \mathrm{keV}$, which allows our telescope to observe sources at lower energies.

The energy resolution does not depend strongly on the selected events class and improves considerably with increasing energy: at $100 \mathrm{keV}$ it is roughly $\sim 20 \%$ while at $\sim 3 \mathrm{MeV}$ it is $3 \%$. At $1 \mathrm{MeV}$, the energy resolution is $4.6 \%$ (FWHM). Notice, however, that above a few MeVs, there are severe issues with incomplete energy absorption due to the limited size of the detector.

To estimate the angular resolution, we compute the ARM and SPD values for tracked and untracked events (see Figure 5). For the ARM, we consider only the full width at half maximum. One should still be careful when using such a value, as it only takes into account the peak of the spectrum and does not include the contributions from the tails of the distribution. However, in the energy range we consider, the ARM distribution is regular enough as to not introduce any major issue due to the presence of significant tails. Similarly, to estimate the SPD, we compute the HWHM of the distribution.

As expected, as we can see in Figure 5, tracked events present generally larger ARM values compared to untracked ones. Nevertheless, for this event class we can compute the SPD, so it can still provide a better total angular resolution despite the broadening of ARM peaks.

Finally, using the semi-analytical method explained, we can also give an estimate of the continuum sensitivity the detector can achieve, visible in Figure 6. We assume an effective observation time of $T_{\text {eff }}=10^{6} \mathrm{~s}$ with the source close to the zenith and a significance level of $z=3$.

As we can see, at lower energies, untracked events achieve a better sensitivity than do tracked ones. This is due to the fact that the effective area for tracked events is extremely small. Therefore, such a telescope should work with untracked events at energies $\lesssim 500 \mathrm{keV}$ and with tracked events at energies $\gtrsim 500 \mathrm{keV}$. However, if we compare the signal-to-noise ratio
$(\mathrm{S} / \mathrm{N})$ of the two classes, we can see that untracked events have a much lower $\mathrm{S} / \mathrm{N}$ (see Figure 7). This implies that systematics will be a major issue.

We also computed the performance for pair production. For the detector under study, pair production plays a role of secondary importance compared to Compton scattering. In the energy range studied (from $\sim 2 \mathrm{MeV}$ up to $\sim 10 \mathrm{MeV}$ ), the pair production cross section is quite small, so we cannot reach a substantial effective area, while at higher energies, we would need much more active material to fully absorb incoming particles. The effective area, the PSF, and the sensitivity estimations can be found respectively in Figures 3, 5, and 6. As we can see, we cannot reach a satisfying performance for pair production with a nano-satellite design.

As a closing remark, we compute the number of expected photon for a source $\left(N_{\mathrm{S}}\right)$. Considering the Crab Nebula spectrum and using tracked events, we estimate to observe $N_{\mathrm{S}} \sim 1.7 \cdot 10^{4}$ photons for an effective observation time of $10^{6} \mathrm{~s}$, with an $\mathrm{S} / \mathrm{N}$ of $\sim 25 \%$. Instead, with untracked events, we estimate $N_{\mathrm{S}} \sim 2.0 \cdot 10^{5}$ photons with an $\mathrm{S} / \mathrm{N}$ of $\sim 6 \%$.

\section{Conclusions}

In this paper, we evaluated the viability and the performance of a nano-satellite Compton telescope. The instrument will operate in the energy range $\sim 100 \mathrm{keV}$ up to few MeVs.

At present, the sensitivity estimated for the designed telescope is comparable to that achieved by COMPTEL at $1 \mathrm{MeV}$. The major problem is the $\mathrm{S} / \mathrm{N}$, which would imply control of systematics at percent level.

Here, we present some possible improvements to the design studied in this paper:

1. Increasing effective area improves the sensitivity, but not the $\mathrm{S} / \mathrm{N}$ (as we also increase the background). Whereas, decreasing the active material to take into account the 


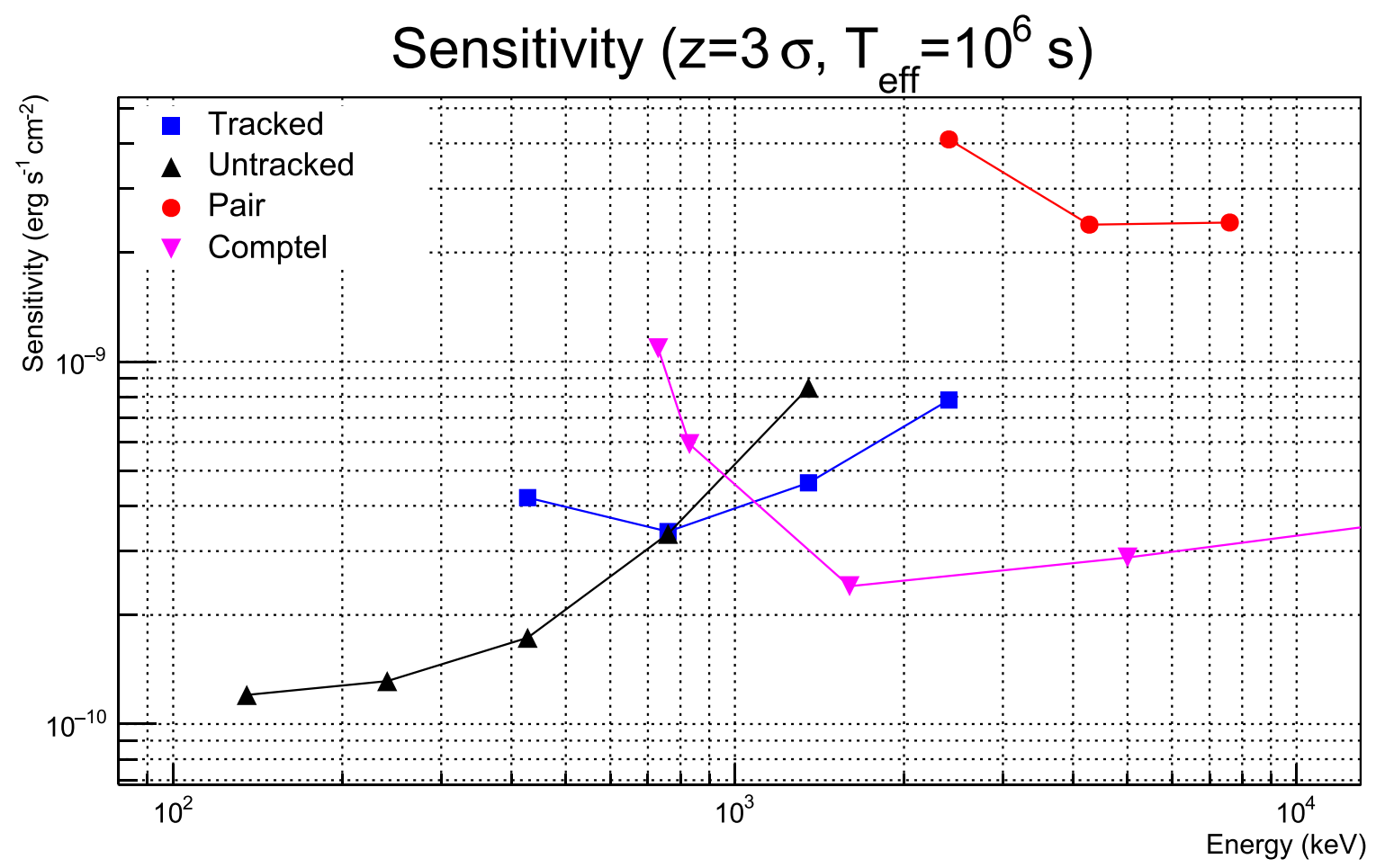

Figure 6. Estimated continuum sensitivity for Compton (both tracked and untracked) and pair events, in comparison to that of COMPTEL. COMPTEL sensitivity values are taken from Schönfelder (2004).

\section{Signal-to-Noise Ratio}

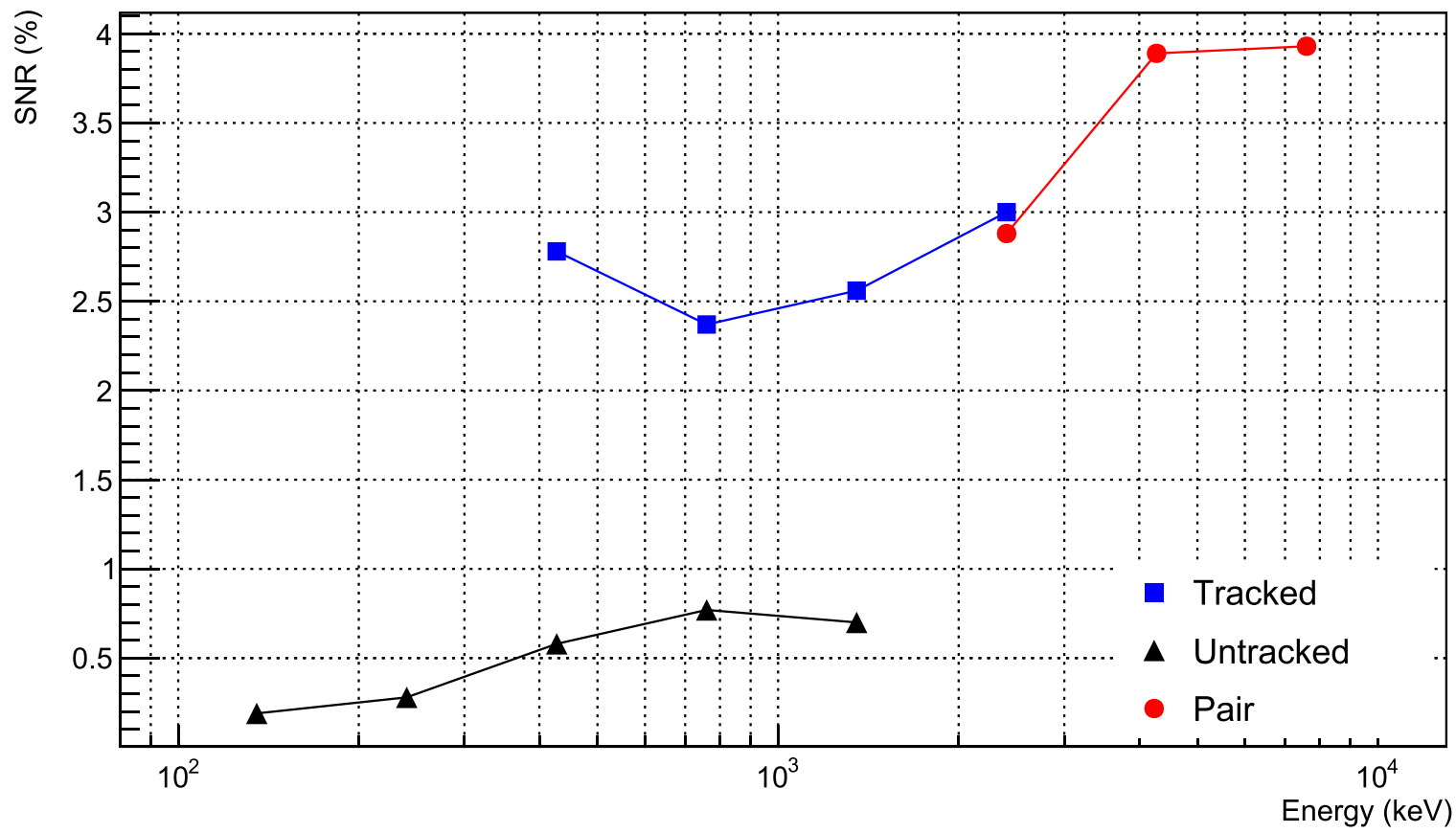

Figure 7. Estimated signal-to-noise ratio for Compton (both tracked and untracked) and pair events.

read-out electronics has a limited influence on the sensitivity $(15 \div 20 \%$ worsening).

2. In our design, angular resolution is quite large, but with our sensitivity, we expect few steady sources $(\sim 30$ as in the COMPTEL catalog, see Schönfelder et al. (2000)). From the results of our simulations, we know that a better depth resolution for the calorimeter crystals yields a significantly better ARM. In fact, with a depth resolution of $0.5 \mathrm{~cm}$, the ARM FWHM is reduced by $\sim 40 \%$ at energies $\sim 1 \mathrm{MeV}$ and normal incidence, which reflects improvements of $\sim 35 \%$ in the sensitivity and $\mathrm{S} / \mathrm{N}$.

3. Earth's gamma emission is reconstructed correctly, but a fraction of events reach the sky map element close to the zenith angle due to the large SPD. Improving the SPD 
enhances the $\mathrm{S} / \mathrm{N}$ through two different contributions: first, we improve the angular resolution element and second, we reject background events. However, SPD values are strongly limited by multiple scattering in silicon; without a substantial redesign of the tracker, we cannot significantly change the performance obtained.

Software: MegaLib (Zoglauer et al. 2008).

\section{References}

Acharya, B. S., Actis, M., Aghajani, T., et al. 2013, APh, 43, 3

Ackermann, M., Ajello, M., Allafort, A., et al. 2013, ApJ, 765, 54

Atwood, W. B., Abdo, A. A., Ackermann, M., et al. 2009, ApJ, 697, 1071

Berlato, F. 2016, MSc thesis, Univ. Padova

Bloser, P. F., Hunter, S. D., Ryan, J. M., et al. 2004, NewAR, 48, 299

Bloser, P. F., Ryan, J. M., McConnell, M. L., et al. 2006, ChJA\&A, 6, 388

Chang, J. 2014, ChJSS, 34, 550
De Angelis, A., Tatischeff, V., Tavani, M., et al. 2017, ExA, in press (arXiv:1611.02232) https://doi.org/10.1007/s10686-017-9533-6

Gehrels, N., Chincarini, G., Giommi, P., et al. 2004, ApJ, 611, 1005

Georg, W. 1999, PhD thesis, Technischen Universität München

Harrison, F. A., Craig, W. W., Christensen, F. E., et al. 2013, ApJ, 770, 103

Horns, D. 2016, in Journal of Physics Conference Ser. 718, ed. N. Fornengo, M. Regis, \& H. Zechlin (Bristol: IOP), 022010

Kappadath, S. C. 1998, PhD thesis, Univ. New Hampshire

Lucchetta, G. 2016, MSc thesis, Univ. Padova

Odaka, H., Ichinohe, Y., Takeda, S., et al. 2012, NIM A, 695, 179

Schönfelder, V. 2004, NewAR, 48, 193

Schönfelder, V., Aarts, H., Bennett, K., et al. 1993, ApJS, 86, 657

Schönfelder, V., Bennett, K., Blom, J. J., et al. 2000, A\&A, 143, 145

Tanimori, T., Kubo, H., Takada, A., et al. 2015, ApJ, 810, 28

Tavani, M., Barbiellini, G., Argan, A., et al. 2008, A\&A, 502, 995

Winkler, C., Diehl, R., Ubertini, P., et al. 2011, SSRv, 161, 149

Zoglauer, A., Andritschke, R., Boggs, S. E., et al. 2008, Proc. SPIE, 7011, $70113 \mathrm{~F}$

Zoglauer, A. C. 2005, PhD thesis, Max-Planck-Institut für Extraterrestrische Physik 